\title{
Amorphous Graphene Paper for Ultrastable Sodium-Ion Batteries \\ Phosphorus/Nitrogen-Doped
}

Chao Zhang, ${ }^{\dagger, \#}$ Xi Wang, ${ }^{*, \$, \dagger, \#}$ Qifeng Liang, ${ }^{+, \#}$ Xizheng Liu, ${ }^{\psi}$ Qunhong Weng, ${ }^{* \dagger}$ Jiangwei Liu, ${ }^{\dagger}$ Yijun Yang, ${ }^{\S}$ Zhonghua Dai, ${ }^{\&}$ Kejian Ding, ${ }^{\S}$ Yoshio Bando, ${ }^{\dagger}$ Jie Tang, ${ }^{\&}$ and Dmitri Golberg*,†

†International Center for Materials Nanoarchitectonics (WPI-MANA), National Institute for Materials Science (NIMS), Namiki 1-1, Tsukuba, Ibaraki 305-0044, Japan

${ }^{\S}$ School of Sciences, Beijing Jiaotong University, Beijing, 100044, P. R. China

Department of Physics, Shaoxing University, Shaoxing, P. R. China

${ }^{\psi}$ Institute for New Energy Materials and Low-Carbon Technologies, School of Materials Science and Engineering, Tianjin University of Technology, Tianjin 300384, P.R. China

${ }^{\&}$ National Institute for Materials Science (NIMS), Sengen 1-2-1, Tsukuba, Ibaraki 305-0047, Japan

\# These authors contributed equally.

\section{Corresponding Authors}

* wangxicas@gmail.com or Wang.Xi2@nims.go.jp, Weng.Qunhong@nims.go.jp, Golberg.Dmitri@nims.go.jp. 


\section{Experimental sections:}

Synthesis of P@GN. Graphene oxide (GO) suspension used in this work was prepared by a modified method. ${ }^{22} 50 \mathrm{mg}$ of graphene oxide was loaded in a ceramic boat in a tube furnace followed by its heat treatment at $600{ }^{\circ} \mathrm{C}$ for $1.5 \mathrm{~h}$ in a gas mixture of $\mathrm{NH}_{3}$ and $\mathrm{Ar}(1: 2 \mathrm{vol} / \mathrm{vol})$ under a total flow rate of $150 \mathrm{ml} / \mathrm{min}$. Commercial red phosphorus was dispersed in water and put into a Teflon-lined stainless autoclave. The autoclave was heated to $200{ }^{\circ} \mathrm{C}$ and maintained for $12 \mathrm{~h}$ to remove surface oxidation. As-prepared N-doped graphene $(\mathrm{GN})$ products were properly mixed together with the pretreated red phosphorus powder, and sealed in an ampoule in an inert Ar atmosphere. The sealed reactors were thermally treated at 500 ${ }^{\circ} \mathrm{C}$ for $1 \mathrm{~h}$ and then at $280{ }^{\circ} \mathrm{C}$ for several hrs in the furnace, before cooling to room temperature naturally. The final product was washed with $\mathrm{CS}_{2}$, water and methanol, and then dried at $60{ }^{\circ} \mathrm{C}$.

Characterization. Transmission electron microscopy (TEM) images were taken with a $300 \mathrm{kV}$ JEOL 3000F microscope. Samples were first dispersed in ethanol and then collected using carbon-film-covered copper grids for analysis. To avoid possible electron beam effects (such as radiolysis or sputtering damage of both Na-containing species and graphene lattice) the beam intensity was minimized. Scanning electronic microscopy (SEM) images were recorded on a Hitachi S4800 electron microscope operating at $15 \mathrm{kV}$. XRD patterns were recorded on a Philips X Pert PRO MPD X-ray diffractometer operated at $35 \mathrm{kV}$ and $45 \mathrm{~mA}$ with $\mathrm{Cu} \mathrm{K \alpha}$ radiation. XPS measurements were carried out on an ESCALab220i-XL spectrometer by using a twin-anode $\mathrm{Al} \mathrm{Ka}(1486.6 \mathrm{eV}) \mathrm{X}$-ray source. All the spectra were calibrated to the 
binding energy of $\mathrm{C} 1 \mathrm{~s}$ peak at $284.6 \mathrm{eV}$. The background pressure was $\sim 3 \times 10^{-7} \mathrm{~Pa}$. Raman spectra were taken on a Horiba Jobin-Yoon T6400 Raman spectrometer.

Electrochemical tests: The electrochemical properties of P@GN and P NCs@G samples were studied using a 2032-type coin cell on a Hokudo Denko Charge/Discharge apparatus. The working electrode was prepared by directly pressing a piece of sample onto the $\mathrm{Cu}$ mesh current collector. Na metal foil was selected as the reference and counter electrode. The mass loading of active material in electrode was $\sim 1-3 \mathrm{mgcm}^{-2}$. The electrolyte was $1 \mathrm{M} \mathrm{NaPF}_{6}$ in ethyl carbonate (EC) and diethyl carbonate (DEC) $(\mathrm{EC}: \mathrm{DEC}=1: 1 \mathrm{in}$ vol/vol). The cells were assembled in a glove box filled with a pure argon gas.

Construction of individual prototype P@GN (P NCs@G)-based SIB. In situ transmission electron microscopy (TEM) observations were conducted in a JEOL-3100 FEF equipped with an Omega filter and a "Nanofactory Instruments" STM-TEM holder. In order to build up the test cell, an individual P@GN or P NCs@G nanosheets were attached to the gold wire, which was then attached to the piezo-manipulator. A small piece of sodium foil was attached to another gold wire as a reference and counter electrode. Before insertion of the holder into the TEM column, a piece of sodium foil covered with a $\mathrm{Na}_{2} \mathrm{O}$ layer was placed on the surface of metal gold tip. Then isolated P@GN or P NCs@G samples were chosen for direct electrochemical tests and under following delicate piezo-driven TEM mechanical manipulations the two electrodes were connected and the probe cell was constructed. The sodiation was carried out at a negative bias in the range of $-2 \mathrm{~V}$ to $0 \mathrm{~V}$ with respect to the Na metal.

DFT calculations. The first principles calculations were carried out using the Vienna $a b$ initio simulation package (VASP), ${ }^{1}$ where projected-augmented-wave (PAW) 
potential was adopted. ${ }^{2,3]}$ The functional of Perdew, Burke, and Ernzerhof (PBE) and the generalized gradient approximation $(\mathrm{GGA})^{4}$ were employed in the calculations. We used a $3 \times 3 \times 1$ mesh in the irreducible Brillouin Zone for structure relaxation and $6 \times 6 \times 1$ mesh for self-consisted calculations. In all the calculations the forces were relaxed to the values lower than $0.02 \mathrm{eV} / \AA$.

References

1. Kresse; G., Furthmüller; J. Phys. Rev. B 1996, 54, 11169-11186.

2. Blöchl; P. E. Phy. Rev. B 1994, 50, $17953-17979$.

3. Kresse; G., Joubert; D. Phys. Rev. B 1999, 59, 1758-1775.

4. Perdew; J. P., Burke; K., Ernzerhof; M. Phys. Rev. Lett. 1996, 77, 3865-3868. 


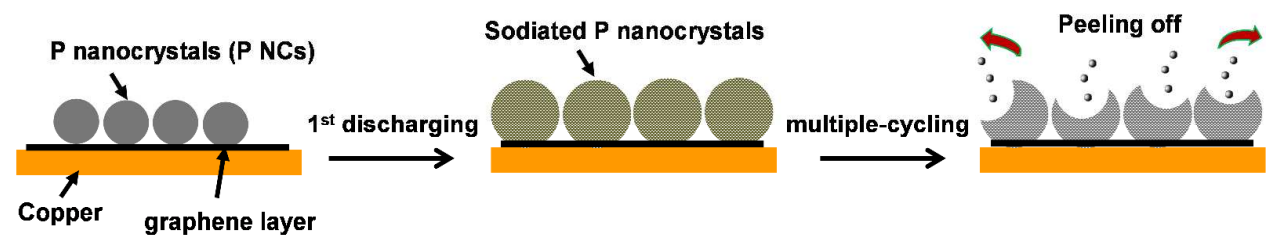

Figure S1. Schematic diagram of the structural fracture of a high-volume-change type $\mathrm{P}$ anode, in which $\mathrm{P}$ nanocrystals anchored on the graphene layer are placed on the surface of a $\mathrm{Cu}$ current collector. After $1^{\text {st }}$ discharging, there is a large volumetric expansion ( $>200-500 \%)$ for P NCs; during cycling, such large volume change will lead to the pulverization and thus peeling off the electrode. 


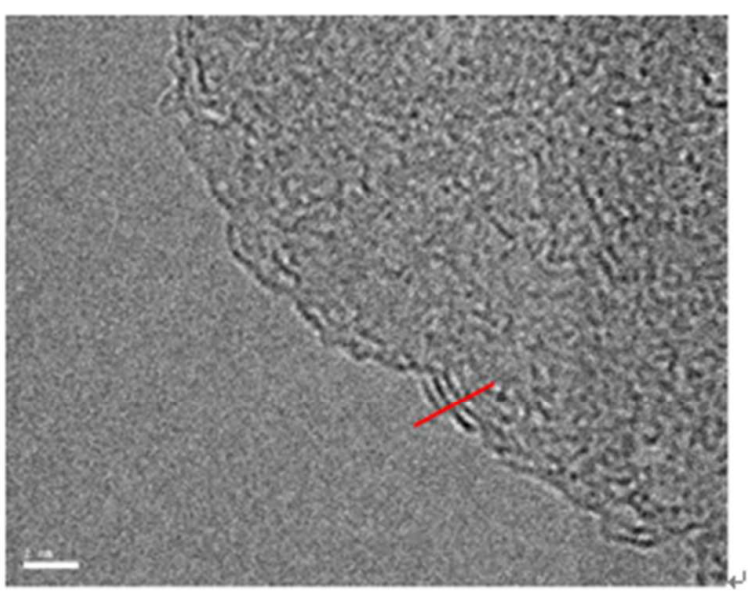

Figure S2. HRTEM image of an N-doped graphene (GN). 

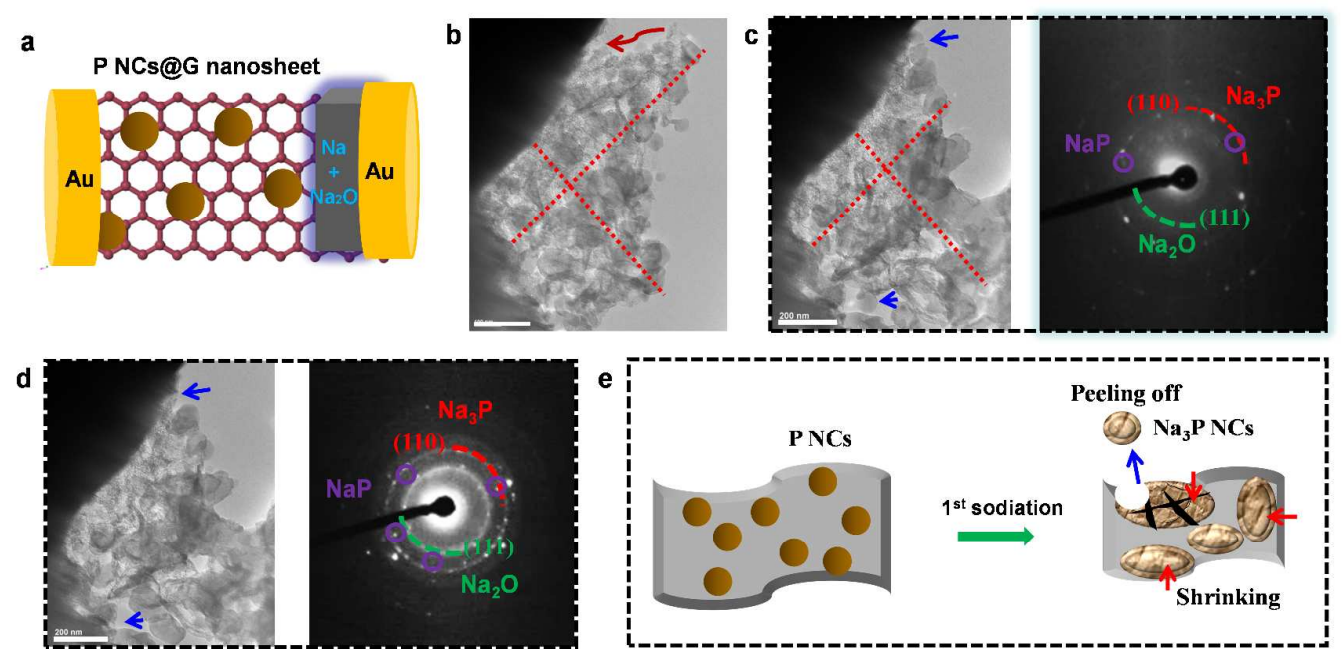

Figure S3. a) Schematic illustration of an individual P nanocrystals@G (P NCs@G) nanosheet prototype sodium battery device fabricated under in situ TEM. b) TEM image of the nano-SIB at the initial stage. c-d) Time-dependent TEM images and selected area electron diffraction (SAED) patterns of sodiated P NCs@G nanosheet upon sodiation: $5 \mathrm{~s}$ and $120 \mathrm{~s}$. The insets $(\mathrm{b}-\mathrm{d})$ show the corresponding schematic structures. Scale bar: $200 \mathrm{~nm}$. e) The schematic illustration of the $1^{\text {st }}$ sodiation-desodiation process of P@GN nanosheet. Red arrows indicate the shrinkage direction during discharging, while blue arrows indicate some particles peeled off during sodiation. 


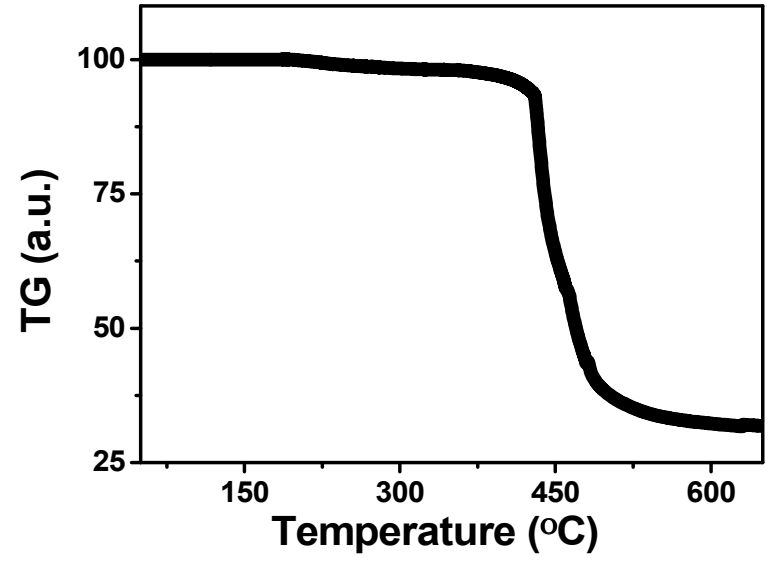

Figure S4. TGA curve of a P@GN sample. 

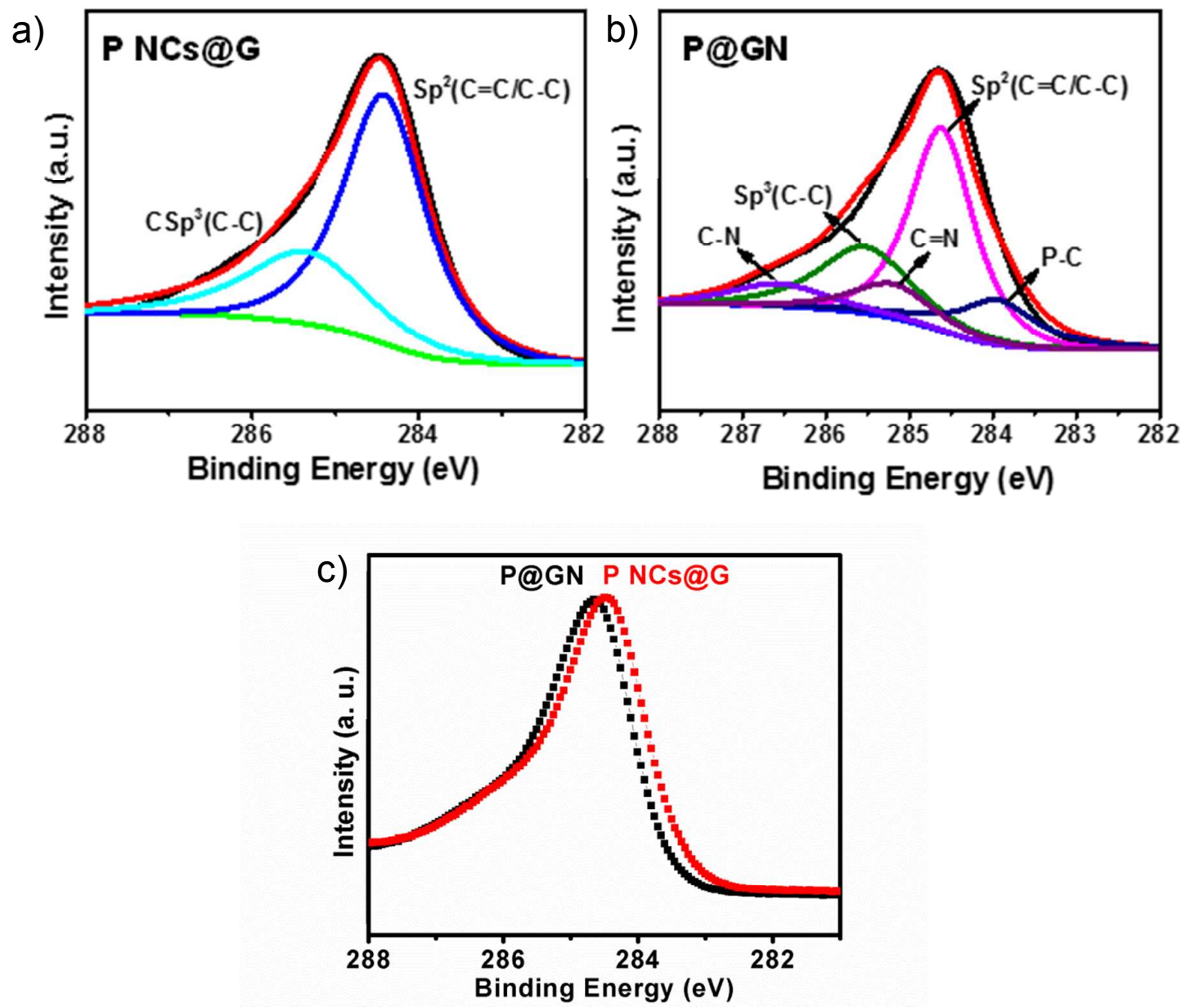

Figure S5. a-b) C1s XPS spectra of PNCs@G and P@GN samples. c) Overlay of the two surveys for $\mathrm{C} 1 \mathrm{~s}$ spectra for the two samples. 

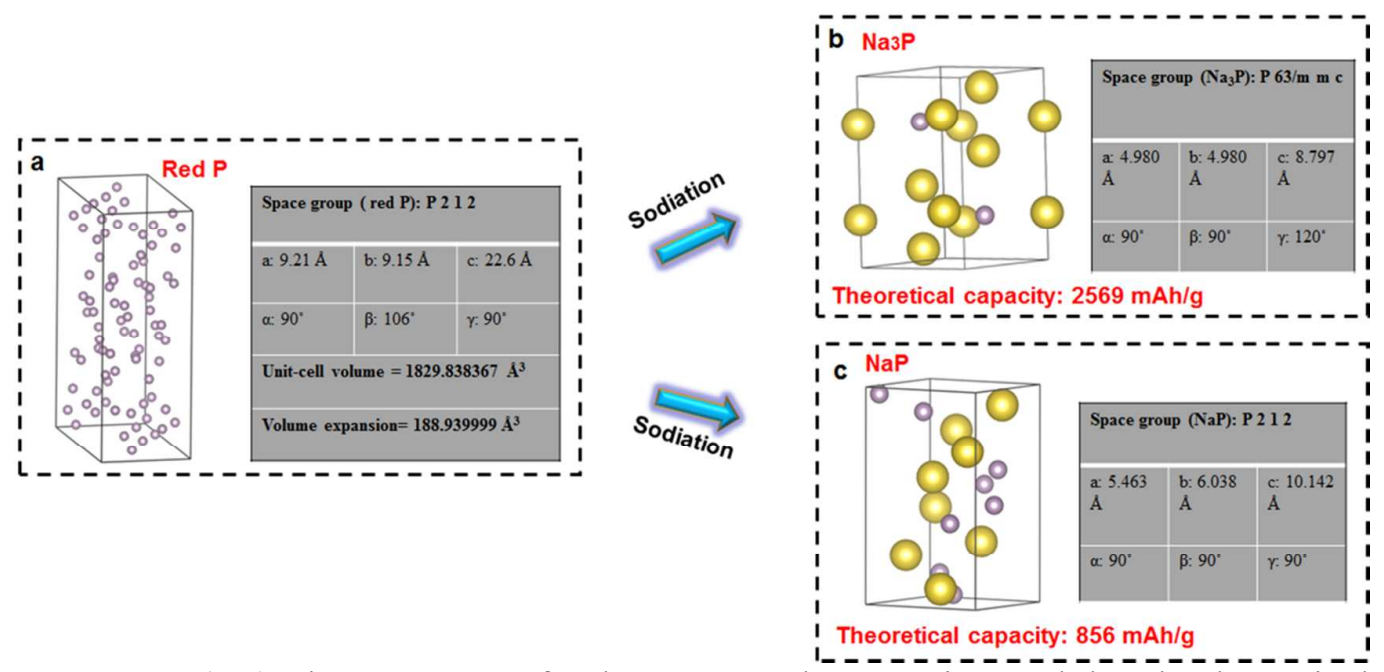

Figure S6. (a-c) The structures of red $\mathrm{P}, \mathrm{Na}_{3} \mathrm{P}$ and $\mathrm{NaP}$. It is noted that the theoretical capacities of $\mathrm{Na}_{3} \mathrm{P}$ and $\mathrm{NaP}$ are different, they are $2569 \mathrm{mAh} / \mathrm{g}$ and $856 \mathrm{mAh} / \mathrm{g}$, respectively. 

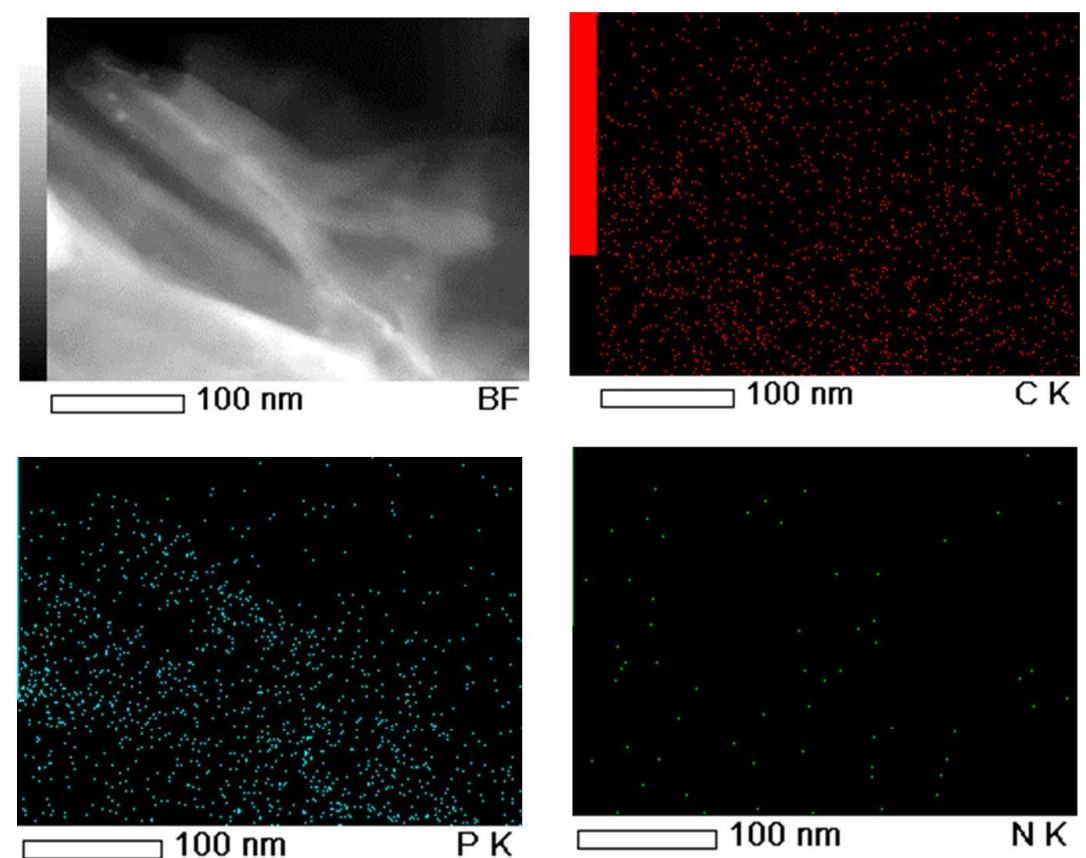

Figure S7. HAADF-STEM image, and C-, P- and N-elemental maps of a P@GN nanosheet. 

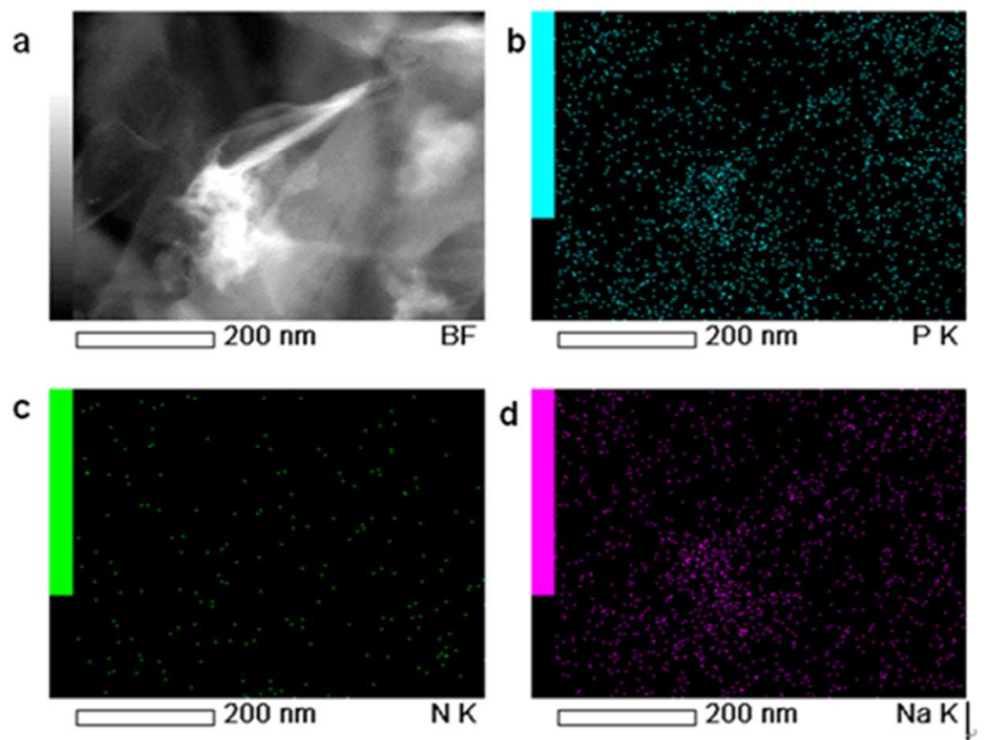

Figure S8. The HAADF-STEM image and the corresponding elemental maps of a P@GN nanosheet at the fully desodiated state after cycling. 
Table S1. Comparison of the charge transfer and theoretical capacity of pure graphene and three different doping graphene in SIBs

\begin{tabular}{lll}
\hline Samples & Pure graphene $(\mathrm{G})$ & $\mathrm{C}_{46} \mathrm{~N}_{3}(\mathrm{GN})$ \\
\hline Charge transfer $(\mathrm{e})$ & - & 0.851 \\
$\begin{array}{l}\text { The calculated } \\
\text { capacity (mAh/g) }\end{array}$ & 0 & 373.3 \\
\hline
\end{tabular}

